\title{
Pérdida de peso y los adultos mayores: Riesgos y beneficios $^{1}$
}

\author{
Wendy Gans, Rachelle Savelle, Nancy Gal, y Wendy J. Dahl; traducido por Daniela \\ Rivero-Mendoza ${ }^{2}$
}

A los adultos obesos a menudo se les aconseja perder peso para reducir el riesgo de enfermedades crónicas (Jensen et al. 2014). Sin embargo, los beneficios para la salud de la pérdida de peso cambian a medida que envejecemos ( $\mathrm{Di}$ Milia, Mittman, and Batsis 2019). Esta publicación analiza los riesgos y beneficios de la pérdida de peso planificada y no planificada para adultos mayores.

La obesidad se asocia con un mayor riesgo de muchas enfermedades crónicas, como enfermedades cardíacas y diabetes tipo 2. La pérdida de peso puede reducir el riesgo de desarrollar enfermedades y afecciones crónicas y mejorar la calidad de vida (Leslie and Hankey 2015). La pérdida de peso también puede facilitar la actividad física. Sin embargo, la "paradoja de la obesidad" describe las observaciones de que cierto exceso de peso puede servir para proteger a los adultos mayores, pero las relaciones entre el peso y los riesgos para la salud son complejas (Bosello and Vanzo 2019). Aunque la obesidad se asocia con una mayor supervivencia de enfermedades graves (Toft-Petersen et al. 2018), los adultos mayores obesos con baja masa muscular tienen un mayor riesgo de malos resultados durante y después de una enfermedad crítica (Tieland, van Dronkelaar, and Boirie 2019). La obesidad puede retrasar la recuperación del accidente cerebrovascular (Kalichman, Alperovitch-Najenson, and Treger 2016), pero en contraste, mejora la recuperación luego de una fractura de cadera (Nishioka et al. 2020).

La obesidad se define como un índice de masa corporal (IMC), mayor de 30, la cual es la relación entre la altura y el peso corporal, El rango de IMC con la mortalidad más baja (riesgo de muerte) es mayor para los adultos mayores en comparación con los adultos más jóvenes. Esto sugiere que los adultos mayores pueden beneficiarse de pesos corporales ligeramente más altos que los adultos más jóvenes (Bhaskaran et al. 2018). Consulte el siguiente enlace para calcular su IMC: https://www.nhlbi.nih.gov/health/ educational/lose_wt/BMI/bmicalc.htm

\section{¿La pérdida de peso es planificada o no planificada?}

Si la pérdida de peso es planificada o no es una consideración importante para un adulto mayor obeso. La pérdida de peso no planificada, conocida como pérdida de peso no intencional, está relacionada con riesgos para la salud. La pérdida involuntaria de peso desde la mediana edad hasta la edad adulta se asocia con un deterioro cognitivo leve (Alhurani et al. 2016). En mujeres mayores, la pérdida de peso involuntaria se asocia con un mayor riesgo de fractura (Compston et al. 2016). La pérdida de peso involuntaria también se asocia con una disminución funcional (Ritchie et al. 2008) y en combinación con baja masa muscular, está relacionada con una mala calidad de vida en adultos

1. Este documento, FSHN20-42s, es uno de una serie de publicaciones del Food Science and Human Nutrition, Servicio de Extensión Cooperativa de la Florida, Instituto de Alimentos y Ciencias Agrícolas, Universidad de la Florida. (UF/IFAS). Fecha de primera publicación: octubre 2020. Visite nuestro sitio web EDIS en https://edis.ifas.ufl.edu.

2. Wendy Gans, former MS-DI student; Rachelle Savelle, former MS-DI student, Food Science and Human Nutrition Department; Nancy J. Gal, Extension agent IV, Food and Consumer Sciences, UF/IFAS Marion County Extension Service, Ocala, FL; and Wendy Dahl, associate professor, Food Science and Human Nutrition Department; traducido por Daniela Rivero Mendoza, Extension and research coordinator; UF/IFAS Extension, Gainesville, FL 32611.

The Institute of Food and Agricultural Sciences (IFAS) is an Equal Opportunity Institution authorized to provide research, educational information and other services

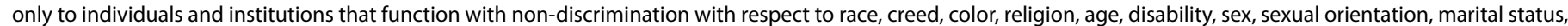

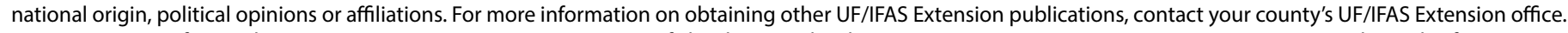
U.S. Department of Agriculture, UF/IFAS Extension Service, University of Florida, IFAS, Florida A \& M University Cooperative Extension Program, and Boards of County Commissioners Cooperating. Nick T. Place, dean for UF/IFAS Extension. 
mayores que viven en la comunidad (Kim, Kim, and Won 2018). La pérdida de peso no planificada significativa puede afectar la supervivencia (Cao, Hardy, and Wulaningsih 2019).

\section{Caso de estudio}

Susan es su vecina de 79 años. Cuando usted le dijo que parecía que había perdido algo de peso, ella respondió felizmente: "iSí, he perdido casi 15 libras!" Susan ha tenido sobrepeso la mayor parte de su vida y ha sido obesa durante los últimos 15 años. ¿La pérdida de peso reciente de Susan es algo bueno?

\section{¿Por qué es común la pérdida de peso involuntaria en adultos}

\section{mayores?}

La pérdida de peso involuntaria a menudo se debe a la reducción de los alimentos como resultado de la falta de apetito. La falta de apetito puede ser consecuencia de enfermedades, medicamentos, soledad o depresión (Gaddey and Holder 2014). Las dificultades para masticar y tragar, debido a sequedad de boca, medicamentos o afecciones como accidente cerebrovascular, demencia o enfermedad neuromuscular, también pueden conducir a una reducción de la ingesta de alimentos y una pérdida de peso involuntaria (Christmas and Rogus-Pulia 2019). El consumo de una dieta con textura modificada puede ayudar a mantener una segura capacidad de tragar e ingerir alimentos, pero puede disminuir la calidad de vida (Swan et al. 2015). Otras barreras para la ingesta adecuada de alimentos en los adultos mayores pueden incluir la falta de dinero para comprar suficientes alimentos nutritivos y dificultades para comprar o preparar alimentos.

Si un adulto mayor pierde peso involuntariamente, debe visitar a un profesional de la salud para determinar la causa de la pérdida de peso y cómo abordarla. Es muy importante que el adulto mayor haga un seguimiento con su proveedor de atención médica, ya que la pérdida de peso puede deberse a una enfermedad no diagnosticada como el cáncer (Bosch et al. 2017).

\section{¿Qué se puede hacer para evitar la pérdida de peso involuntaria?}

Es importante que los adultos mayores disfruten de una alta calidad de vida, puedan realizar actividades de la vida diaria y mantengan su independencia. Cuando se produce una pérdida de peso involuntaria, el adulto mayor y su equipo de atención médica deben desarrollar estrategias para identificar y manejar los problemas relacionados con la pérdida de peso involuntaria. Un equipo integral de profesionales de la salud puede incluir un dietista, médico, terapeuta ocupacional, fisioterapeuta, patólogo del habla y el lenguaje, dentista y trabajador social. Las adaptaciones nutricionales y de estilo de vida pueden mejorar la ingesta dietética y evitar una mayor pérdida de peso involuntaria. A continuación hay algunos consejos para ayudar a prevenir la pérdida de peso involuntaria, especialmente la pérdida muscular.

Consejos para ayudar a prevenir la pérdida de peso.

- Consuma las calorías adecuadas para satisfacer las necesidades energéticas.

- Consuma más alimentos proteicos como carne, pollo, mariscos, huevos y lácteos.

- Consuma más proteínas de origen vegetal como la soya, las legumbres, los frutos secos y las semillas.

- Elija alimentos lácteos enteros en lugar de lácteos bajos en grasa.

- Consuma refrigerios (bocadillos o tentempiés) o suplementos nutricionales entre comidas.

- Discuta con su proveedor de atención médica sobre cualquier problema de masticación y problemas para tragar.

- Discuta con su proveedor de atención médica los riesgos y beneficios de cualquier restricción en su dieta.

El nutricionista dietista registrado (RDN, por sus siglas en inglés) es el especialista en nutrición del equipo de atención médica. Para los adultos mayores, los RDN brindan un servicio vital al proporcionar terapias de nutrición médica necesarias para controlar las condiciones de salud existentes. Los RDN ayudan a promover una nutrición óptima para evitar la pérdida de peso involuntaria y mejorar la calidad de vida.

\section{Pérdida de peso intencional}

La pérdida de peso planificada puede tener beneficios para algunos adultos mayores (Wannamethee, Shaper, and Lennon 2005). Si un adulto mayor con obesidad planea bajar de peso, debe hacerlo bajo la supervisión de su proveedor de atención médica. La forma más segura y efectiva de perder peso es combinando un patrón de alimentación saludable y un programa de ejercicio aprobado por un médico. Esto conducirá a un mayor bienestar cardiovascular, fuerza muscular y masa corporal magra (libre de grasa), al tiempo que minimiza el riesgo de desnutrición que puede ocurrir al reducir las calorías. La pérdida de peso no debe buscarse de manera insegura eliminando grupos de alimentos o siguiendo una dieta de moda. 
La mejor manera de lograr la pérdida de peso es gradualmente, con la salud y la calidad de vida generales del individuo como prioridad. Siempre consulte con su proveedor de atención médica antes de comenzar una dieta o un programa de actividad física. El siguiente sitio web proporciona información confiable sobre la actividad física y el envejecimiento: https://www.nia.nih.gov/health/ exercise-physical-activity.

Entonces, ¿se debería felicitar a Susan por su pérdida de peso? Es importante determinar si la pérdida de peso de Susan fue involuntaria o planificada. En cualquier caso, Susan debe ver a su proveedor de atención médica y elaborar un plan para minimizar cualquier riesgo potencial para la salud relacionado con la pérdida de peso. Susan debe centrarse en un estilo de vida saludable que mejore su bienestar general.

\section{Referencias}

Alhurani, R. E., M. Vassilaki, J. A. Aakre, M. M. Mielke, W. K. Kremers, M. M. Machulda, Y. E. Geda, D. S. Knopman, R. C. Petersen, and R. O. Roberts. 2016. "Decline in Weight and Incident Mild Cognitive Impairment: Mayo Clinic Study of Aging." JAMA Neurol 73 (4): 439-46. doi: 10.1001/ jamaneurol.2015.4756.

Bhaskaran, K., I. Dos-Santos-Silva, D. A. Leon, I. J. Douglas, and L. Smeeth. 2018. "Association of BMI with Overall and Cause-Specific Mortality: A PopulationBased Cohort Study of 3.6 Million Adults in the UK." Lancet Diabetes Endocrinol 6 (12): 944-953. doi: 10.1016/ s2213-8587(18)30288-2.

Bosch, X., E. Monclús, O. Escoda, M. Guerra-García, P. Moreno, N. Guasch, and A. López-Soto. 2017. "Unintentional Weight Loss: Clinical Characteristics and Outcomes in a Prospective Cohort of 2677 Patients." PLoS One 12 (4): e0175125. doi: 10.1371/journal.pone.0175125.

Bosello, O., and A. Vanzo. 2019. "Obesity Paradox and Aging.” Eat Weight Disord. doi: 10.1007/s40519-019-00815-4.

Cao, Y., R. Hardy, and W. Wulaningsih. 2019. "Associations of Medical Conditions, Lifestyle and Unintentional Weight Loss in Early Old Age: The 1946 British Birth Cohort." PLoS One 14 (4): e0211952. doi: 10.1371/journal.pone.0211952.

Christmas, C., and N. Rogus-Pulia. 2019. "Swallowing Disorders in the Older Population." J Am Geriatr Soc 67 (12): 2643-2649. doi: 10.1111/jgs.16137.

Compston, J. E., A. Wyman, G. FitzGerald, J. D. Adachi, R. D. Chapurlat, C. Cooper, A. Díez-Pérez, S. H. Gehlbach, S. L. Greenspan, F. H. Hooven, A. Z. LaCroix, L. March, J.
C. Netelenbos, J. W. Nieves, J. Pfeilschifter, M. Rossini, C. Roux, K. G. Saag, E. S. Siris, S. Silverman, N. B. Watts, and F. A. Anderson, Jr. 2016. "Increase in Fracture Risk Following Unintentional Weight Loss in Postmenopausal Women: The Global Longitudinal Study of Osteoporosis in Women." J Bone Miner Res 31 (7): 1466-72. doi: 10.1002/jbmr.2810.

DiMilia, P. R., A. C. Mittman, and J. A. Batsis. 2019. "Benefit-to-Risk Balance of Weight Loss Interventions in Older Adults with Obesity." Curr Diab Rep 19 (11): 114. doi: 10.1007/s11892-019-1249-8.

Gaddey, H. L., and K. Holder. 2014. "Unintentional Weight Loss in Older Adults." Am Fam Physician 89 (9): 718-22.

Jensen, M. D., D. H. Ryan, C. M. Apovian, J. D. Ard, A. G. Comuzzie, K. A. Donato, F. B. Hu, V. S. Hubbard, J. M. Jakicic, R. F. Kushner, C. M. Loria, B. E. Millen, C. A. Nonas, F. X. Pi-Sunyer, J. Stevens, V. J. Stevens, T. A. Wadden, B. M. Wolfe, and S. Z. Yanovski. 2014. "2013 AHA/ACC/TOS Guideline for the Management of Overweight and Obesity in Adults: A Report of the American College of Cardiology/ American Heart Association Task Force on Practice Guidelines and The Obesity Society." J Am Coll Cardiol 63 (25 Pt B): 2985-3023. doi: 10.1016/j.jacc.2013.11.004.

Kalichman, L., D. Alperovitch-Najenson, and I. Treger. 2016. “The Impact of Patient's Weight on Post-Stroke Rehabilitation." Disabil Rehabil 38 (17): 1684-90. doi: 10.3109/09638288.2015.1107640.

Kim, M., J. Kim, and C. W. Won. 2018. "Association Between Involuntary Weight Loss with Low Muscle Mass and Health-Related Quality of Life in CommunityDwelling Older Adults: Nationwide Surveys (KNHANES 2008-2011)." Exp Gerontol 106:39-45. doi: 10.1016/j. exger.2018.02.027.

Leslie, W., and C. Hankey. 2015. "Aging, Nutritional Status and Health." Healthcare (Basel) 3 (3): 648-58. doi: 10.3390/ healthcare3030648.

Nishioka, S., H. Wakabayashi, K. Maeda, H. Shamoto, Y. Taketani, J. Kayashita, and R. Momosaki. 2020. "Body Mass Index and Recovery of Activities of Daily Living in Older Patients with Femoral Fracture: An Analysis of a National Inpatient Database in Japan." Arch Gerontol Geriatr 87:104009. doi: 10.1016/j.archger.2020.104009.

Ritchie, C. S., J. L. Locher, D. L. Roth, T. McVie, P. Sawyer, and R. Allman. 2008. "Unintentional Weight Loss Predicts Decline in Activities of Daily Living Function 
and Life-space Mobility over 4 Years among CommunityDwelling Older Adults." J Gerontol A Biol Sci Med Sci 63 (1): 67-75. doi: 10.1093/gerona/63.1.67.

Swan, K., R. Speyer, B. J. Heijnen, B. Wagg, and R. Cordier. 2015. "Living with Oropharyngeal Dysphagia: Effects of Bolus Modification on Health-Related Quality of Life-A Systematic Review." Qual Life Res 24 (10): 2447-56. doi: 10.1007/s11136-015-0990-y.

Tieland, M., C. van Dronkelaar, and Y. Boirie. 2019. "Sarcopenic Obesity in the ICU." Curr Opin Clin Nutr Metab Care 22 (2): 162-166. doi: 10.1097/mco.0000000000000547.

Toft-Petersen, A. P., J. Wulff, D. A. Harrison, M. Ostermann, M. Margarson, K. M. Rowan, and D. Dawson. 2018. "Exploring the Impact of Using Measured or Estimated Values for Height and Weight on the Relationship between BMI and Acute Hospital Mortality." J Crit Care 44:196-202. doi: 10.1016/j.jcrc.2017.11.021.

Wannamethee, S. G., A. G. Shaper, and L. Lennon. 2005. "Reasons for Intentional Weight Loss, Unintentional Weight Loss, and Mortality in Older Men." Arch Intern Med 165 (9): 1035-40. doi: 10.1001/archinte.165.9.1035. 